\title{
A Three-Market Comparative Study on Determinants of Firm Performance: The Commercial Banking Industry
}

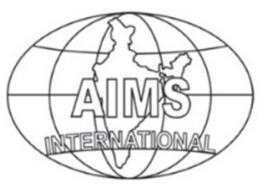

DOI: $10.26573 / 2018.12 .2 .2$

Volume 12, Number 2

May 2018, pp. 99-119

\author{
Tao (Tony) Gao \\ University of Massachusetts Lowell \\ (tao_gao@uml.edu) \\ James P. Neelankavil \\ K. G. Viswanathan \\ Hofstra University \\ (james.neelankavil@gmail.com) \\ (K.G.Viswanathan@hofstra.edu)
}

Past research on bank performance has mostly examined determinants of bank profitability in either domestic (such as the U.S. or U.K.) or single-region (such as Europe or Latin America) markets. A key point of departure for the current study is the explanation of performance differences among commercial banks from multiple regions (North America, Asia, and Europe). With data covering over twenty years from more than two hundred commercial banks in three large industrialized countries (U.S., Japan, and Italy), we seek to identify key firm, industry, and country drivers for bank performance as commercial banks compete on global versus domestic markets. We further use both profitability and growth performance measures to seek to uncover unique determinants of each type of measure in a global banking environment.

Keywords: Determinants of Bank Performance, Bank Performance, Bank Profitability, Bank Growth, Global Competition, Commercial Banks, CrossCountry Studies

\section{Introduction}

Commercial banks from major industrialized countries such as Canada, France, Germany, Italy, Japan, the United Kingdom, and the United States compete in a worldwide market for a share of the banking business. Yet, their global expansion is largely dependent on how well they are established in their domestic markets. Hence, banks such as JP Morgan Chase (U.S.), Deutsche Bank (Germany), HSBC (U.K.), and Sumitomo (Japan) all have a major share of the domestic banking market while posting strong showings globally. In such a global banking environment, it is useful to understand how these banks perform against one another and how the key drivers of superior performance change as banks move from domestic to global markets.

Past studies have predominantly examined determinants of bank profitability in either domestic (such as the U.S., Greece, Canada, and UAE) or single-region (such as Europe) markets. Some of these studies suggest that bank-specific factors such as capital structure, credit risk, productivity, and operating expenses influence profitability (Fadzlan and Majid, 2007; Bodla and Verma, 2006; Kosmidou et al., 2006; On gore, 2013). Others demonstrate the importance of macroeconomic and other external factors as determinants of bank profits ( $\mathrm{Wu}$, Chen, and Lin, 2007; 
Chirwa, 2003; Yildrim, 2002; Cornett, Ors, and Tehranian, 2002). At the same time, a few studies have shown the importance of both internal and external factors in influencing profits (Athanasoglou, Brissims, and Delis, 2008; Naceur and Goaied, 2001; Fraser, Phillips, and Rose, 1974; Degl'Innocenti et al., 2017; Bucevska and Misheva, 2017).

A similar pattern is observed in cross-country, regional studies of banking performance. Some studies show that internal factors are the driving force of profits (e.g., Al-Tamimiand Lootah, 2007; Goddard, Molyneux, and Wilson, 2004), while others note the significance of external factors (Bourke, 1989). Still more studies conclude that it is both the internal and external factors that are critical in determining profits when comparing banks across countries (Barros, Ferreira and Williams, 2007; De Guevara and Maudos, 2002; Demirguc-Kuntand Huizinga, 1998; Short, 1979).

A key point of departure for the current study is the more global focus. With multi-region data covering over twenty years from more than two hundred commercial banks in three large industrialized countries (U.S., Japan, and Italy), we seek to identify key firm, industry, and country drivers for bank performance as commercial banks compete on global as opposed to domestic markets. We further use both profitability and growth performance measures to uncover unique determinants of each type of measure in a global banking environment.

Specifically, our study seeks to contribute to the existing literature on bank performance by addressing the following research questions

1. What are notable differences in commercial banks' performance across three different regions?

2. What are key firm, industry, and country determinants of cross-regional differences in bank performance? Answers to this question will help enhance the understanding of what it takes for commercial banks to succeed globally.

3. What are the key differences in performance drivers as banks move from domestic competition to global competition? Answers to this question will help banks prepare for the successful transition from domestic competition to global competition.

4. What are key differences in determinants of bank performance across profit and growth measures? This question serves to verify the robustness of our findings and explore the unique determinants of profit and growth performance.

In the rest of the paper, we review the prior literature on bank performance, describe our methodology, report and discuss our key results, and discuss the research and managerial implications of our study.

\section{Literature Review}

A bank's performance can be measured using an assortment of variables including profitability ratios, total revenues, and sales growth (Alam, 2003; Goddard, Molyneux, and Wilson, 2004). Most existing studies of bank performance have studied determinants of bank profitability and the two most frequently used profitability ratios are return on asset (ROA) and return on equity (ROE) (Athanasoglou et al., 2008; Chirwa, 2003; Kosmidou et al., 2006; Seemule, Sinha and Ndlovu, 2017). 
A fundamental issue in analyzing bank profitability is whether superior profitability is driven by internal factors, external factors, or both. Studies dealing with internal determinants consider the impacts on bank performance by such variables as size, capital or capital-asset ratios, ownership, risk management, employee productivity, and expense or cost management. For example, in analyzing operational efficiencies (operational expenses over revenues or similar measures), researchers have found that their impact on bank performance is sometimes influenced by other external factors such as industry structure, macroeconomic conditions, and regulations (Al-Tamimi and Liotah, 2007; Canhoto and Dermine, 2003; Isik and Hassan, 2002). Accounting ratios such as debt-to-equity ratio have also been shown to impact a bank's performance (Goddard, Molyneux, and Wilson, 2004). Similarly, a bank's capital-asset ratios (e.g., equity/assets) positively affect performance (Athanasoglou et al., 2008).

Other variables that have some effects on a bank's performance are type of ownership (Short, 1979) and bank size (Barros, Ferreira, and Williams, 2007). For example, Griffith, Fogelberg, and Weeks (2002) focus specifically on how CEO ownership influences bank performance as measured by market value added (MVA), economic value added (EVA), and Tobin's $q$ and, interestingly, note a negative relationship between CEO ownership and bank performance. Research findings on banks' relative size suggest that the size of a bank helps enhance its performance due to the scale economy (Barros, Ferreira, and Williams, 2007; Goddard, Molyneux, and Wilson, 2004).

Research studies also find that risk is highly correlated with bank profitability in that banks holding less-risky assets are more profitable than those holding high-risk assets (Yildirim and Philippatos, 2007; Gintschelandn Hackethal, 2004; Bourke, 1989). Other internal factors including operational or cost management are found to influence profit efficiency (Barros, Ferreira, and Williams, 2007; Fraser, Phillips, and Rose, 1974). Employee productivity has also been found to positively correlate with performance in two single-country studies (Athanasoglou et al., 2008; Mehra, 1996). In contrast, a few other studies note an opposite relationship between employee productivity and bank performance in that the best-performing banks were those that struggle to improve labor and capital productivity (Gintschel and Hackethal, 2004; Naceur and Goaied, 2001). As can be seen from the preceding discussions, many of the internal variables do not consistently affect bank performance and various studies have revealed opposite effects.

External determinants of bank profitability can be divided further into macroeconomic variables and market-related variables. Macroeconomic variables include GDP growth rate, inflation rate, cyclical output, and interest rate, while market characteristics refer to market concentration, industry size, and ownership status.

Several studies show a positive relationship between GDP growth rate and bank performance (Staikouras, 2005; Goddard, Molyneux, and Wilson, 2004; Shehzad, de Haan and Scholtens, 2013; Heggestad, 1977). Additionally, changes in inflation and cyclical output are known to affect banking performance (Athanasoglou et al., 2008). As expected, higher inflation has a negative effect on banking performance, and moderate or lower inflation has a positive effect on performance. Goddard, Molyneux, and Wilson (2004) show that a bank's performance is influenced by liquidity ratio, Herfindahl and Hirschman index, (HHI), and GDP growth. At the 
same time, Quagliariello (2009), Wan et al. (2008), and Yildirim (2002) find that macroeconomic uncertainty and expansion or contraction have significant bearings on banks' investment and expansion decisions, their efficiency and profitability. Surprisingly, very few studies have considered interest rates as a variable that might affect banking performance.

External variables related to market characteristics such as market concentration, industry size, and ownership status may affect banking performance as well (Bucevska and Misheva 2017). For example, Bourke (1989) finds that higher concentration in banking markets encourages banks to hold less-risky assets and, hence, safeguard their profitability. Similarly, Heggestad (1977) and Fraser, Phillips, and Rose (1974) find that the structure of banking markets moderately affects banking performance. In contrast, Yildirim and Philippatos (2007) conclude that concentration in the banking industry does not necessarily lead to a lower level of competition and higher bank performance.

\section{Methodology}

Data for the proposed study were obtained from the database DataStream, which contains financial data for more than one hundred countries and many industries including banking. Commercial banking data for three industrialized countries Japan, Italy, and the United States - were collected. Originally, about ten countries were considered for the study, but an exploratory review of the data revealed discrepancies and missing data for seven of the ten countries. We chose for the final study three countries for which full financial information was available for at least twenty-five commercial banks. The combined data set is an unbalanced panel of 4,326 observations of 207 banks (90 U.S., 90 Japanese, and 27 Italian) over a twenty-one-year period (1985 - 2005). Compustat data were also utilized for some of the measures. The International Monetary Fund's World Economic Outlook and International Financial Statistics databases were employed for measures such as GDP growth rates and fund rates for the three countries.

For this study, we decided to exclude data for 2006 and beyond to avoid any distortions directly attributed to the financial crisis of 2008. While many industries worldwide were adversely affected by the crisis, the banking industry was affected the most and central banks across the world had to implement various strategies over several years following the crisis to stabilize the borrowing and lending functions in individual markets. The distortions caused by the crisis and the responses from central banks to mitigate the adverse effects were not uniform across countries and regions. Consequently, in this study, we avoid the confounding effects by excluding banks in the sample for the two years leading up to the crisis and the years following the crisis.

\subsection{Dependent Variables}

In analyzing the data, we chose the following performance variables as the dependent variables: return on assets (ROA), return on equity (ROE), and sales growth. These variables have been used in previous bank performance studies (Mansur and Elyasiani, 1995; Strischek, 1987; Hartman, 2006; Sensarma, 2006). ROA indicates the ability of the bank to earn satisfactory returns on an important item of its balance sheet, essentially measuring the overall returns on its investments. Researchers have consistently used ROA as a measure in conducting bank 
performance studies, especially to measure divisional and corporate performance after the initial investments are completed (Wu, Chen, and Lin, 2007; Reilly and Wright, 2004; Jensen and Meckling, 2009). Return on equity (ROE) indicates return on the owner's investment in the firm. Since ROE focuses on the shareholders, it is considered a crucial measure of a firm's performance. Research has shown that there exists a high correlation between higher growth in ROE and earnings per share (Kumar and Sopariwala, 1992). Research studies have also shown that there is a positive stock market reaction to long-term performance plans as well as subsequent higher growth rates for those firms that have high ROE (Ferris, et al., 1998). The last performance measure, sales growth, reflects the ability of the firm to maintain and grow its revenue (Bodla and Verma, 2006).

\subsection{Independent Variables}

We attempted to study banking performance both within and in cross-country settings using some of the most commonly used independent variables in major bank performance studies (cf. Athanasoglou et al., 2008; Al-Tamimiand Lootah, 2007; Bodla and Verma, 2006; Canhoto and Dermine, 2003; Fadzlanand Majid, 2007; Goddard, Molyneux, and Wilson, 2004; Isik and Hassan, 2002; Kosmidou et al., 2006; Naceur and Goaied, 2001). They include balance sheet structure variables such as liquidity [loans/assets (L/A)], capitalization [common equity/assets (CE/A)], and debt leverage [debt/equity (D/E)]; and firm operational variables such as revenue diversification [interest income/net sales (II/NS)], operating efficiency [operating expenses/net sales (OE/NS)], asset turnover [net sales/assets (NS/A)], and interest spread ratio or gross margin [net interest income/interest income (NII/INCOME)].

To account for cross-national differences in bank operating environments, we used GDP growth rate, fund rate, and a commonly used measure of industry concentration, the Herfindahl-Hirschman Index (HHI), as country-level explanatory variables (Mehra, 2006; Park and Weber, 2006; Chine-Ta, 2006).

\subsection{Control Variables}

Our main control variable in each of the regression equations was the dependent variable as measured in the previous year. It has the advantage of being comprehensive (Nagar and Rajan, 2005) and its inclusion allows us to test the persistency of good (or bad) performance in each measure and competitive setting. Furthermore, using only this measure keeps the number of regressor's low, an important consideration given the large number of independent variables. The sensitivity analyses demonstrate the robustness of our results to the inclusion of additional control variables. To allow for comparison with previous studies that used various specific bank sizes and capacity controls (e.g., Yildirim and Philippatos, 2007), we also use assets and number of employees as firm-level control variables.

\subsection{Descriptive Statistics for Banks}

\section{Analyses}

Table 1 presents the number of banks and descriptive statistics of bank characteristics for each country. All data are reported in U.S. dollars as the reference currency and adjusted for inflation. The large standard deviations for the variables indicate a substantial amount of variance in the responses. 
Table 1 Descriptive Statistics of the Data Set and ANOVA Results for Cross-Country Differences in Study Variables (1985-2005)

\begin{tabular}{|c|c|c|c|c|c|c|c|c|c|}
\hline \multirow{2}{*}{ Variables } & \multicolumn{2}{|c|}{ All 3 Countries } & \multicolumn{2}{|c|}{ United States } & \multicolumn{2}{|c|}{ Japan } & \multicolumn{2}{|c|}{ Italy } & \multirow{2}{*}{$\begin{array}{l}\text { ANOVA of } \\
\text { Means } \\
\text { (F Value) }\end{array}$} \\
\hline & Mean & SD & Mean & SD & Mean & SD & Mean & SD & \\
\hline ROA (\%) & 0.63 & 1.50 & 1.11 & 1.36 & 0.07 & 0.46 & 0.73 & 3.10 & $219.86^{*}$ \\
\hline ROE (\%) & 8.95 & 14.34 & 15.62 & 7.73 & 1.63 & 17.53 & 8.14 & 6.96 & $474.41 *$ \\
\hline Sales Growth (\%) & 4.63 & 15.34 & 9.41 & 14.61 & -1.04 & 13.55 & 6.03 & 17.56 & $196.62 *$ \\
\hline LY ROA (\%) & 0.62 & 1.53 & 1.10 & 1.39 & 0.06 & 0.46 & 0.73 & 3.12 & $199.91^{*}$ \\
\hline LY ROE (\%) & 8.89 & 14.40 & 15.63 & 7.82 & 1.46 & 17.61 & 8.03 & 6.94 & $457.92 *$ \\
\hline $\begin{array}{l}\text { LY Sales Growth } \\
(\%)\end{array}$ & 4.33 & 15.29 & 8.86 & 14.40 & -1.23 & 13.76 & 6.14 & 17.52 & $174.75^{*}$ \\
\hline
\end{tabular}

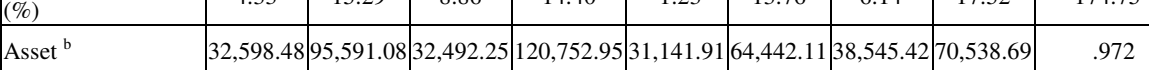

\begin{tabular}{|l|l|l|l|l|l|l|l|l|r|}
\hline Employees & $5,961.58$ & $17,194.28$ & $8,278.67$ & $23,670.50$ & $2,537.88$ & $2,659.33$ & $9,486.94$ & $15,228.14$ & $54.82 *$ \\
\hline
\end{tabular}

\begin{tabular}{|l|c|c|c|c|c|c|c|c|c|}
\hline GDP Growth (\%) & 2.59 & 1.72 & 3.10 & 1.21 & 2.33 & 2.07 & 1.80 & 1.31 & $178.37^{*}$ \\
\hline Fund Rate (\%) & 4.38 & 3.38 & 5.11 & 2.22 & 2.46 & 2.62 & 8.29 & 4.34 & $1,091.55^{*}$ \\
\hline Loans/Assets & 0.65 & 0.11 & 0.62 & 0.12 & 0.69 & 0.07 & 0.63 & 0.12 & $183.51^{*}$ \\
\hline $\begin{array}{l}\text { Common } \\
\text { Equity/Assets }\end{array}$ & 0.07 & 0.05 & 0.08 & 0.04 & 0.04 & 0.01 & 0.09 & 0.12 & $368.25^{*}$ \\
\hline $\begin{array}{l}\text { II/NS (Interest } \\
\text { Weight) }\end{array}$ & 0.82 & 0.12 & 0.82 & 0.12 & 0.84 & 0.11 & 0.76 & 0.12 & $75.73^{*}$ \\
\hline Debt/Equity & 186.64 & $1,091.02$ & 202.85 & 189.40 & 47.33 & $1,641.06$ & 638.42 & 428.23 & $48.72^{*}$ \\
\hline NII/INCOME & 0.54 & 0.21 & 0.53 & 0.14 & 0.57 & 0.28 & 0.42 & 0.14 & $74.04^{*}$ \\
\hline NS/A & 0.08 & 0.12 & 0.10 & 0.17 & 0.04 & 0.01 & 0.10 & 0.10 & $108.21^{*}$ \\
\hline OE/NS & .30 & .22 & .24 & .12 & .40 & .29 & .21 & .10 & $309.17^{*}$ \\
\hline $\begin{array}{l}\text { Number of } \\
\text { Observations }\end{array}$ & \multicolumn{2}{|c|}{3,046} & \multicolumn{2}{|c|}{1,409} & 1,327 & & 310 & \\
\hline Number of Banks & \multicolumn{2}{|c|}{207} & 90 & & 27 & \\
\hline
\end{tabular}

Notes: a: Sample means are for Bank-Year Observations. b: In Thousands of U.S. Dollars.

$*$ Represents Significance at $p=.001$

\subsection{Cross-National Differences in Bank Performance}

We estimated the cross-country differences in the three bank performance measures with a MANOVA test. In doing so, we included country as the independent variable, all three performance measures as the dependent variables, and year as the control variable. The results of this test, as presented in Table 2, show significant crosscountry differences in bank performance regardless of the measure used. These differences are indicated by the significance of both Wilks' lambda for the global MANOVA and $F$ values associated with each test in the MANOVA. Results from a follow-up ANOVAs, t-tests, and discriminant analyses show that for all 3 measures, U.S. banks significantly outperform Italian banks, and U.S. and Italian banks significantly outperform Japanese banks.

\subsection{Correlation Analysis}

We first examined the relationship between various explanatory variables and bank performance by investigating simple inter-variable correlation coefficients for each 
country. The values displayed in Tables 3 - 5 feature both significant and nonsignificant correlations between study variables, providing an initial test of the effects of various determinants on bank performance in each country. For example, the correlation coefficients show the importance of previous year's performance and $\mathrm{OE} / \mathrm{NS}$ as strong predictors, and asset as a relative non-factor, in influencing bank performance.

Table 2 MANOVA Analysis of Cross-Country Differences in Bank Performance

\begin{tabular}{|l|c|c|c|c|c|}
\hline \multicolumn{1}{|c|}{ Variables } & All 3 Countries & US & Japan & Italy & F Value \\
\hline ROA & 0.63 & 1.11 & 0.07 & 0.73 & $488.37 *$ \\
\hline ROE & 8.95 & 15.62 & 1.63 & 8.14 & $455.42 *$ \\
\hline Sales Growth (\%) & 4.63 & 9.41 & -1.04 & 6.03 & $195.14 *$ \\
\hline Number of Observations & 3,046 & 1,409 & 1,327 & 310 & \\
\hline Number of Banks & 207 & 90 & 90 & 27 & \\
\hline $\begin{array}{l}\text { Wilks' Lambda for } \\
\text { MANOVA }\end{array}$ & \multicolumn{7}{|c|}{$236.32 *$} \\
\hline
\end{tabular}

Notes: *: Significant at $p=.001$. Sample means were for all Bank-Year Observations

Table 3 Intercorrelations of Study Variables-United States

\begin{tabular}{|l|c|c|c|c|c|c|c|c|c|c|c|c|c|c|c|c|c|c|}
\hline \multicolumn{1}{|c|}{ Variables } & 1 & 2 & 3 & 4 & 5 & 6 & 7 & 8 & 9 & 10 & 11 & 12 & 13 & 14 & 15 & 16 & 17 & 18 \\
\hline 1. ROA & 1.00 & & & & & & & & & & & & & & & & & \\
\hline 2. ROE & 0.35 & 1.00 & & & & & & & & & & & & & & & & \\
\hline 3. Sales Growth & 0.09 & 0.26 & 1.00 & & & & & & & & & & & & & & & \\
\hline 4. LYROA & 0.22 & 0.33 & 0.10 & 1.00 & & & & & & & & & & & & & & \\
\hline 5. LY ROE & $\mathbf{0 . 0 2}$ & 0.54 & 0.21 & 0.34 & 1.00 & & & & & & & & & & & & & \\
\hline 6. LY Sales Growth & $\mathbf{0 . 0 2}$ & 0.19 & 0.31 & 0.09 & 0.26 & 1.00 & & & & & & & & & & & & \\
\hline 7. Asset & $\mathbf{0 . 0 0}$ & 0.08 & $\mathbf{0 . 0 3}$ & $\mathbf{0 . 0 1}$ & 0.09 & $-\mathbf{0 . 0 1}$ & 1.00 & & & & & & & & & & & \\
\hline 8. Employees & $\mathbf{0 . 0 1}$ & 0.10 & $\mathbf{0 . 0 1}$ & $\mathbf{0 . 0 1}$ & 0.10 & $\mathbf{0 . 0 1}$ & 0.96 & 1.00 & & & & & & & & & & \\
\hline 9. GDP Growth & 0.06 & 0.09 & 0.24 & $\mathbf{0 . 0 4}$ & $\mathbf{0 . 0 3}$ & $\mathbf{0 . 0 4}$ & $\mathbf{0 . 0 1}$ & $-\mathbf{- 0 . 0 1}$ & 1.00 & & & & & & & & & \\
\hline 10. Fund Rate & -0.09 & -0.06 & 0.31 & -0.06 & $\mathbf{- 0 . 0 3}$ & 0.36 & -0.15 & -0.13 & 0.19 & 1.00 & & & & & & & & \\
\hline 11. HHI & 0.09 & 0.09 & 0.05 & 0.09 & 0.09 & -0.10 & 0.07 & 0.05 & 0.11 & -0.54 & 1.00 & & & & & & & \\
\hline 12. L/A & 0.12 & $\mathbf{0 . 0 1}$ & 0.07 & 0.11 & $\mathbf{0 . 0 2}$ & 0.08 & -0.22 & -0.21 & $\mathbf{0 . 0 3}$ & $\mathbf{0 . 0 1}$ & 0.04 & 1.00 & & & & & & \\
\hline 13. CE/A & 0.25 & -0.12 & -0.07 & 0.26 & -0.19 & -0.09 & -0.06 & -0.07 & $\mathbf{0 . 0 1}$ & -0.11 & 0.13 & 0.14 & 1.00 & & & & & \\
\hline 14.II/NS & -0.12 & -0.16 & 0.08 & -0.13 & -0.13 & 0.12 & -0.30 & -0.32 & $\mathbf{0 . 0 2}$ & 0.33 & -0.14 & 0.24 & -0.05 & 1.00 & & & & \\
\hline 15. D/E & -0.07 & 0.06 & 0.08 & -0.06 & 0.09 & $\mathbf{0 . 0 5}$ & 0.20 & 0.25 & $\mathbf{0 . 0 5}$ & $-\mathbf{0 . 0 3}$ & $\mathbf{0 . 0 1}$ & $\mathbf{0 . 0 4}$ & -0.25 & -0.14 & 1.00 & & & \\
\hline 16. NII/INCOME & 0.28 & 0.14 & -0.15 & 0.26 & 0.09 & -0.26 & $\mathbf{0 . 0 2}$ & $\mathbf{0 . 0 1}$ & $\mathbf{0 . 0 1}$ & -0.67 & 0.42 & $-\mathbf{- 0 . 0 3}$ & 0.39 & -0.20 & -0.40 & 1.00 & & \\
\hline 17. NS/A & 0.24 & -0.20 & $-\mathbf{0 . 0 2}$ & 0.13 & -0.27 & $\mathbf{- 0 . 0 3}$ & $\mathbf{- 0 . 0 3}$ & $-\mathbf{0 . 0 3}$ & $\mathbf{0 . 0 1}$ & 0.10 & $-\mathbf{- 0 . 0 4}$ & $\mathbf{0 . 0 3}$ & 0.84 & -0.23 & -0.08 & -0.44 & 1.00 & \\
\hline 18. OE/NS & -0.64 & -0.46 & -0.17 & -0.53 & -0.38 & -0.15 & -0.23 & -0.20 & $-\mathbf{0 . 0 5}$ & $-\mathbf{- 0 . 0 2}$ & $\mathbf{0 . 0 4}$ & $\mathbf{0 . 0 2}$ & $-\mathbf{0 . 0 5}$ & 0.40 & -0.31 & 0.25 & -0.19 & 1.00 \\
\hline
\end{tabular}

Note: All Correlations were Significant at $p=.05$ except those Shown in Bold Font. 
Table 4 Intercorrelations of Study Variables-Japan

\begin{tabular}{|c|c|c|c|c|c|c|c|c|c|c|c|c|c|c|c|c|c|c|}
\hline Variables & 1 & 2 & 3 & 4 & 5 & 6 & 7 & 8 & 9 & 10 & 11 & 12 & 13 & 14 & 15 & 16 & 17 & 18 \\
\hline 1. ROA & 1.00 & & & & & & & & & & & & & & & & & \\
\hline 2. ROE & 0.42 & 1.00 & & & & & & & & & & & & & & & & \\
\hline 3. Sales Growth & 0.08 & 0.17 & 1.00 & & & & & & & & & & & & & & & \\
\hline 4. LY ROA & 0.08 & 0.02 & 0.08 & 1.00 & & & & & & & & & & & & & & \\
\hline 5. LY ROE & -0.05 & 0.40 & 0.10 & 0.43 & 1.00 & & & & & & & & & & & & & \\
\hline 6. LY Sales Growth & -0.01 & 0.00 & -0.03 & 0.08 & 0.17 & 1.00 & & & & & & & & & & & & \\
\hline 7. Asset & -0.06 & 0.12 & -0.07 & -0.06 & 0.12 & -0.09 & 1.00 & & & & & & & & & & & \\
\hline 8. Employees & -0.07 & 0.09 & -0.04 & -0.07 & 0.12 & -0.06 & 0.96 & 1.00 & & & & & & & & & & \\
\hline 9. GDP Growth & 0.11 & 0.09 & 0.21 & 0.04 & 0.11 & -0.20 & -0.14 & -0.08 & 1.00 & & & & & & & & & \\
\hline 10. Fund Rate & 0.01 & -0.02 & 0.28 & 0.03 & 0.02 & 0.21 & -0.23 & -0.15 & 0.41 & 1.00 & & & & & & & & \\
\hline 11. HHI & 0.06 & 0.22 & 0.20 & 0.01 & 0.09 & 0.00 & 0.11 & 0.05 & 0.14 & 0.33 & 1.00 & & & & & & & \\
\hline 12. L/A & -0.07 & -0.03 & -0.08 & -0.19 & -0.11 & -0.10 & 0.12 & 0.12 & -0.26 & -0.44 & -0.38 & 1.00 & & & & & & \\
\hline 13. CE/A & 0.53 & 0.01 & -0.01 & 0.59 & 0.00 & -0.01 & -0.17 & -0.20 & 0.10 & 0.13 & 0.12 & -0.35 & 1.00 & & & & & \\
\hline 14.II/NS & -0.20 & -0.15 & 0.10 & -0.03 & -0.04 & 0.12 & -0.06 & -0.03 & 0.13 & 0.48 & 0.16 & -0.10 & -0.27 & 1.00 & & & & \\
\hline 15. D/E & -0.31 & -0.09 & -0.02 & -0.20 & -0.19 & 0.00 & 0.36 & 0.39 & -0.08 & -0.21 & -0.17 & 0.32 & -0.38 & 0.01 & 1.00 & & & \\
\hline 16. NII/INCOME & 0.16 & 0.16 & -0.10 & 0.08 & 0.13 & -0.12 & 0.01 & -0.14 & -0.24 & -0.64 & -0.29 & 0.24 & 0.08 & -0.39 & -0.23 & 1.00 & & \\
\hline 17. NS/A & -0.02 & -0.13 & 0.02 & 0.02 & -0.11 & 0.00 & -0.15 & -0.14 & 0.00 & 0.19 & 0.02 & -0.39 & 0.13 & 0.22 & -0.23 . & -0.48 & 1.00 & \\
\hline 18. OE/NS & -0.44 & -0.43 & -0.15 & -0.21 & -0.18 & -0.01 & -0.07 & -0.09 & -0.14 & -0.29 & -0.37 & 0.17 & -0.10 & 0.21 . & -0.01 & 0.56 & -0.25 & 1.00 \\
\hline
\end{tabular}

Note: All Correlations were Significant at $p=.05$ except those Shown in Bold Font

Table 5 Intercorrelations of Study Variables-Italy

\begin{tabular}{|c|c|c|c|c|c|c|c|c|c|c|c|c|c|c|c|c|c|c|}
\hline Variables & 1 & 2 & 3 & 4 & 5 & 6 & 7 & 8 & 9 & 10 & 11 & 12 & 13 & 14 & 15 & 16 & 17 & 18 \\
\hline 1. $\mathrm{ROA}$ & 1.00 & & & & & & & & & & & & & & & & & \\
\hline 2. ROE & 0.42 & 1.00 & & & & & & & & & & & & & & & & \\
\hline 3. Sales Growth & 0.08 & 0.17 & 1.00 & & & & & & & & & & & & & & & \\
\hline 4. LY ROA & 0.08 & 0.02 & 0.08 & 1.00 & & & & & & & & & & & & & & \\
\hline 5. LY ROE & -0.05 & 0.40 & 0.10 & 0.43 & 1.00 & & & & & & & & & & & & & \\
\hline 6. LY Sales Growth & -0.01 & 0.00 & -0.03 & 0.08 & 0.17 & 1.00 & & & & & & & & & & & & \\
\hline 7. Asset & -0.06 & 0.12 & -0.07 & -0.06 & 0.12 & \begin{tabular}{|l|}
-0.09 \\
\end{tabular} & 1.00 & & & & & & & & & & & \\
\hline 8. Employees & -0.07 & 0.09 & -0.04 & -0.07 & 0.12 & -0.06 & 0.96 & 1.00 & & & & & & & & & & \\
\hline 9. GDP Growth & 0.11 & 0.09 & 0.21 & 0.04 & 0.11 & -0.20 & -0.14 & \begin{tabular}{|l|}
-0.08 \\
\end{tabular} & 1.00 & & & & & & & & & \\
\hline 10. Fund Rate & 0.01 & -0.02 & 0.28 & 0.03 & 0.02 & 0.21 & -0.23 & -0.15 & 0.41 & 1.00 & & & & & & & & \\
\hline 11. HHI & 0.06 & 0.22 & 0.20 & 0.01 & 0.09 & 0.00 & 0.11 & 0.05 & 0.14 & 0.33 & 1.00 & & & & & & & \\
\hline 12. $\mathrm{L} / \mathrm{A}$ & -0.07 & -0.03 & -0.08 & -0.19 & -0.11 & -0.10 & 0.12 & 0.12 & -0.26 & -0.44 & -0.38 & 1.00 & & & & & & \\
\hline 13. $\mathrm{CE} / \mathrm{A}$ & 0.53 & 0.01 & -0.01 & 0.59 & 0.00 & -0.01 & -0.17 & -0.20 & 0.10 & 0.13 & 0.12 & -0.35 & 1.00 & & & & & \\
\hline $14 . \mathrm{II} / \mathrm{NS}$ & -0.20 & -0.15 & 0.10 & -0.03 & -0.04 & 0.12 & -0.06 & -0.03 & 0.13 & 0.48 & 0.16 & -0.10 & -0.27 & 1.00 & & & & \\
\hline 15. D/E & -0.31 & -0.09 & -0.02 & -0.20 & -0.19 & 0.00 & 0.36 & 0.39 & -0.08 & -0.21 & -0.17 & 0.32 & -0.38 & 0.01 & 1.00 & & & \\
\hline 16. NII/INCOME & 0.16 & 0.16 & -0.10 & 0.08 & 0.13 & -0.12 & 0.01 & -0.14 & -0.24 & -0.64 & -0.29 & 0.24 & 0.08 & -0.39 & -0.23 & 1.00 & & \\
\hline 17. NS/A & -0.02 & -0.13 & 0.02 & 0.02 & -0.11 & 0.00 & -0.15 & -0.14 & 0.00 & 0.19 & 0.02 & -0.39 & 0.13 & 0.22 & -0.23 & -0.48 & 1.00 & \\
\hline 18. OE/NS & -0.44 & -0.43 & -0.15 & -0.21 & -0.18 & -0.01 & -0.07 & -0.09 & -0.14 & -0.29 & -0.37 & 0.17 & -0.10 & 0.21 & -0.01 & 0.56 & -0.25 & 1.00 \\
\hline
\end{tabular}

Note: All Correlations were Significant at $p=.05$ except those Shown in Bold Font. 
4.4 Model Testing

We examined the associations between the independent variables and bank performance through a series of regressions, as detailed below

$Y=\beta_{0}+\beta_{1} X_{1}+\beta_{2} X_{2}+\beta_{3} X_{3}+\beta_{4} X_{4}+\beta_{5} X_{5}+\beta_{6} X_{6}+\beta_{7} X_{7}+\beta_{7} X_{7}+\beta_{8} X_{8}+\beta_{9} X_{9}+$ $\beta_{10} X_{10}+\beta_{11} X_{11}+\beta_{12} X_{12}+\beta_{13} X_{13}+\xi$

Where

$\mathrm{Y}=$ Bank performance at year $\mathrm{t}$, as measured by ROA, ROE, and sales growth, respectively

$\beta_{0=}$ Constant term

$\beta_{1}-\beta_{13}=$ Regression parameters for the respective variables

$\mathrm{X}_{1}=$ Bank performance at year $\mathrm{t}-1$ using the same measure as the dependent variable

$\mathrm{X}_{2}=$ Asset

$\mathrm{X}_{3}=\quad$ Employees

$\mathrm{X}_{4}=\quad$ GDP growth rate

$\mathrm{X}_{5}=\quad$ Fund rate

$\mathrm{X}_{6}=\quad$ Herfindahl-Hirschman Index

$\mathrm{X}_{7}=\quad$ Loans/assets (L/A)

$\mathrm{X}_{8}=\quad$ Common equity / assets $(\mathrm{CE} / \mathrm{A})$

$\mathrm{X}_{9}=\quad$ Interest weight (interest income / net sales) (II/NS)

$\mathrm{X}_{10}=$ Debt/equity (D/E)

$\mathrm{X}_{11}=$ Interest spread ratio (net interest income / interest income) (NII/INCOME)

$\mathrm{X}_{12}=$ Net sales $/$ assets (NS/A)

$\mathrm{X}_{13}=\quad$ Operating expenses / net sales (OE/NS)

$\xi=\quad$ Error term

We tested for violations of standard regression assumptions concerning model misspecification using Ramsey's (1969) RESET test, normality using the JarqueBera test, and heteroskedasticity using the Breusch-Pagan test. None of these violations appear to be either generalized or problematic in our data.

Time-series cross-sectional data sets also present the potential for problems associated with serial correlation. Failure to account for such problems can lead to biased estimates (particularly of the standard errors) and therefore can result in inefficient estimates (Kennedy, 2003). Durbin-Watson statistics (Kennedy, 2003) suggested that there is no problem of serial correlation in the time-series data utilized for the study, as the value obtained by Durbin-Watson test was around 2 in all regressions. Given these results, we performed fixed-effects regression analyses as our main method for testing effects of the determinant variables on bank performance.

For robustness check, we also employed GLS fixed-effects estimators, as Hausman tests (Boulding and Stalin, 1995; Greene, 2003) indicated that fixed-effect corrections are necessary for our regressions. Because we were already controlling for a variety of firm and country covariates, we accomplished this by introducing year-specific dummies in our analyses (Kennedy, 2003). None of the year dummy variables were found to be significant in any of our regressions. We found qualitatively similar results (which are not reported, to save space). We also used the 
logarithmic transformation method to transform certain independent and control variables to rerun the analyses, and no major differences were noted in doing so. Collectively, these sensitivity procedures ensure the robustness of the results reported in Tables 6-8.

In addition, an examination of the correlation matrix revealed relatively high correlations between some independent and control variables, such as between $\mathrm{OE} / \mathrm{NS}$ and NII/INCOME, and between fund rate and NII/INCOME. These incidences of high bivariate correlations might lead to potential multicollinearity problems in subsequent regression analyses if these variables are entered into the same regression equation. A precautionary step was taken to avoid the multicollinearity problem in each regression. Specifically, we used the stepwise regression approach to reduce the impacts on estimated parameter caused by nonsignificant variables. In addition to inter-variable correlations, condition indices, variance proportions, and variance inflation factors were jointly monitored to inform the analytical process. As a result of these efforts, the results reported in Tables 6-8 were free of impact by multicollinearity, illustrated by all condition indices being below 30 and all variance inflation factors being less than 10 (Belsley, Kuh, and Welsch, 1990).

\section{Results}

For each performance measure, we ran an integrative regression analysis for all banks combined and then a separate regression analysis for banks in each country. This allowed us to identify determinants of bank performance for both individual countries and across countries/regions. Previous studies of bank performance such as Barros, Ferreira, and Williams (2007), Goddard, Molyneux, and Wilson (2004), Bourke (1989), and Short (1979) have also studied across country and within country comparisons. Tables $6-8$ present the standardized regression coefficients for our analytical models. The $\mathrm{R}^{2} \mathrm{~s}$ ranged from .22 to .85 , showing relatively high explanatory power of our regression models.

Table 6 Fixed-Effects Multiple Regression Analysis of Determinants of Bank Performance:

$$
R O A
$$

\begin{tabular}{|l|c|c|c|c|}
\hline & All-Countries & United States & Japan & Italy \\
\hline Last Year Control & & & & \\
\hline LY's ROA & $0.24 * * *$ & $0.07 * * *$ & $.10^{* * * *}$ & $0.18^{* * *}$ \\
\hline Firm Size Control & & & & \\
\hline Asset & $-0.04 * *$ & & $-0.05^{* *}$ & \\
\hline Employees & $0.05^{* * *}$ & & & \\
\hline Country-Level Determinants & & & & \\
\hline GDP Growth & $0.08^{* * *}$ & & $0.12^{* * *}$ & $0.06 *$ \\
\hline Fund Rate & $-0.06^{* * *}$ & $0.08^{* * *}$ & & $-0.17 * *$ \\
\hline HHI & & $0.05 * * *$ & $0.06^{* *}$ & $0.09 * *$ \\
\hline Firm Operational Variables & & & & \\
\hline L/A & $-0.01 *$ & & $-0.06^{* * *}$ & $-0.11 * * *$ \\
\hline
\end{tabular}




\begin{tabular}{|l|c|c|c|c|}
\hline CE/A & $0.15 * * *$ & $0.10 * * *$ & $0.12 * * *$ & $0.24 * * *$ \\
\hline II/NS & $0.22 * * *$ & $0.47 * * *$ & $0.36 * * *$ & $0.48 * * *$ \\
\hline D/E & $0.02 * *$ & & $0.07 * * *$ & $0.17 * *$ \\
\hline NII/INCOME & $0.62 * * *$ & $0.75 * * *$ & $0.85 * * *$ & $0.83 * * *$ \\
\hline NS/A & $0.27 * * *$ & $0.26 * * *$ & & $0.16 * * *$ \\
\hline OE/NS & $-0.75 * * *$ & $-0.95 * * *$ & $-1.23 * * *$ & $-0.81 * * *$ \\
\hline Observations & 3330 & 1539 & 1438 & 353 \\
\hline $\mathbf{R}^{2}$ & 0.85 & 0.85 & 0.67 & 0.75 \\
\hline Largest Condition Index & 24.80 & 27.43 & 24.98 & 28.33 \\
\hline
\end{tabular}

Note: Only Significant Results Are Reported. *,**, *** Represent Significance (One-Sided) at $p=.05, p=.01$, and $p=.001$, Respectively

Table 7 Fixed-Effects Multiple Regression Analysis of Determinants of Bank Performance: $R O E$

\begin{tabular}{|c|c|c|c|c|}
\hline & All-Countries & United States & Japan & Italy \\
\hline \multicolumn{5}{|l|}{ Last Year Control } \\
\hline LY's ROE & $0.12 * * *$ & $0.19 * * *$ & $0.06 * *$ & $0.18^{* * *}$ \\
\hline \multicolumn{5}{|l|}{ Firm Size Control } \\
\hline \multicolumn{5}{|l|}{ Asset } \\
\hline \multicolumn{5}{|l|}{ Employees } \\
\hline \multicolumn{5}{|c|}{ Country-Level Determinants } \\
\hline GDP Growth & $0.07 * * *$ & & $0.11 * * *$ & \\
\hline Fund Rate & & $0.13 * * *$ & & $-0.12 *$ \\
\hline HHI & & $0.05 * *$ & & $0.10^{*}$ \\
\hline \multicolumn{5}{|l|}{ Firm Operational Variables } \\
\hline L/A & $-0.06^{* * *}$ & $-0.03 *$ & $-0.04 *$ & $-0.15^{* * *}$ \\
\hline $\mathrm{CE} / \mathrm{A}$ & $-0.13 * * *$ & $-0.34 * * *$ & $0.05 *$ & $-0.28 * * *$ \\
\hline II/NS & $0.28 * * *$ & $0.50 * * *$ & $0.27 * * *$ & $0.47 * * *$ \\
\hline $\mathrm{D} / \mathrm{E}$ & $0.05^{* * *}$ & & $0.05 * *$ & \\
\hline NII/INCOME & $0.72 * * *$ & $0.78 * * *$ & $0.74 * * *$ & $0.83 * * *$ \\
\hline NS/A & $0.20 * * *$ & $0.21 * * *$ & & \\
\hline OE/NS & $-0.97 * * *$ & $-0.96 * * *$ & $-1.02 * * *$ & $-0.87 * * *$ \\
\hline Observations & 3208 & 1492 & 1382 & 334 \\
\hline $\mathbf{R}^{2}$ & 0.58 & 0.63 & 0.45 & 0.54 \\
\hline Largest Condition Index & 22.48 & 25.69 & 28.46 & 26.90 \\
\hline
\end{tabular}

Note: Only Significant Results are Reported. *,**, *** Represent Significance (One-Sided) at $p=.05, p=.01$, and $p=.001$, Respectively 
Table 8 Fixed-Effects Multiple Regression Analysis of Determinants of Bank Performance: Sales Growth

\begin{tabular}{|c|c|c|c|c|}
\hline & All-Countries & United States & Japan & Italy \\
\hline \multicolumn{5}{|l|}{ Last Year Control } \\
\hline LY's Sales Growth & $0.14 * * *$ & $0.18 * * *$ & $0.10 * * *$ & \\
\hline \multicolumn{5}{|l|}{ Firm Size Control } \\
\hline \multicolumn{5}{|l|}{ Asset } \\
\hline \multicolumn{5}{|l|}{ Employees } \\
\hline \multicolumn{5}{|c|}{ Country-Level Determinants } \\
\hline GDP Growth & $0.18 * * *$ & $0.10 * * *$ & & $0.15 * *$ \\
\hline Fund Rate & $0.60 * * *$ & $0.63 * * *$ & $1.11 * * *$ & $0.46 * * *$ \\
\hline HHI & & $0.16 * * *$ & $-0.12 * * *$ & \\
\hline \multicolumn{5}{|c|}{ Firm Operational Determinants } \\
\hline $\mathrm{L} / \mathrm{A}$ & $0.03 *$ & $0.05 *$ & $-0.06 * *$ & \\
\hline $\mathrm{CE} / \mathrm{A}$ & $-0.06 * *$ & $-0.06^{*}$ & $-0.05 * *$ & $-0.18 * * *$ \\
\hline II/NS & $-0.07 * * *$ & $-0.07 *$ & $-0.28 * * *$ & \\
\hline $\mathrm{D} / \mathrm{E}$ & & $0.09 * *$ & & \\
\hline NII/INCOME & $0.34 * * *$ & $0.23 * * *$ & $0.96 * * *$ & $0.36 * * *$ \\
\hline NS/A & $-0.12 * * *$ & $-0.22 * * *$ & $0.25 * * *$ & \\
\hline $\mathrm{OE} / \mathrm{NS}$ & $-0.22 * * *$ & $-0.16 * * *$ & $-0.14 * * *$ & $-0.33 * * *$ \\
\hline Observations & 3053 & 1410 & 1333 & 310 \\
\hline $\mathbf{R}^{2}$ & 0.35 & 0.27 & 0.53 & 0.22 \\
\hline Largest Condition Index & 27.42 & 25.85 & 26.13 & 26.55 \\
\hline
\end{tabular}

Note: Only Significant Results Are Reported. *, **, *** Represent Significance (One-Sided) at $p=.05, p=.01$, and $p=.001$, Respectively

\subsection{Key Determinants of Bank Performance in Cross-Regional Comparisons}

As shown in the first column of Table 6, the differences in ROA among all U.S., Japanese, and Italian banks can be best explained by the following variables: operational expenses/net sales (parameter or $\beta=-.75 ; \mathrm{p}<.001$ ), interest spread ratio (NII/INCOME) $(\beta=.62 ; \mathrm{p}<.001)$, net sales/asset $(\beta=.27 ; \mathrm{p}<.001)$, last year's performance $(\beta=.24 ; \mathrm{p}<.001)$, interest income/net sales (II/NS) $(\beta=.22 ; \mathrm{p}<.001)$, common equity/assets $(\beta=.15 ; \mathrm{p}<.001)$, GDP growth $(\beta=.08 ; \mathrm{p}<.001)$, fund rate $(\beta=-.06 ; p<.001)$, number of employees $(\beta=.05 ; p<.001)$, asset size $(\beta=-.04 ; p$ $<.01)$, and debt/equity $(\beta=.02 ; \mathrm{p}<.01)$. The results for ROE were also similar except for the reversal of the effect of common equity/asset and the lack of significance for asset, employee size, and fund rate (see the first column of Table 7). Based on these results, banks' cross-regional profitability performance as measured by ROA and ROE, two most critical performance measures, are affected by multiple factors including bank operational variables such as operating efficiency, debt/equity, product diversification, and productivity; balance sheet structure 
variables such as common equity/assets; and external factors such as GDP and fund rate. Our results also show a strong positive effect of last year's performance on bank profitability as measured by both ROA and ROE, thus confirming the profitability persistence phenomenon in a cross-regional setting (cf. Goddard, Molyneux, and Wilson, 2004; Nagar and Rajan, 2005). Collectively, these results show what it takes for firms to achieve superior profitability performance in the global banking environment.

The finding that external variables such as GDP and fund rate affect bank performance across the three regions adds important insight to the bank performance literature. While previous research has shown the effects of external variables such as inflation rate and cyclical output on bank profitability (Athanasoglou et al., 2008), the use of fund rate and GDP as determinant variables is a unique feature of our study as this combination has not been studied previously. In fact, prior singlecountry and within-regional studies (e.g., Stubbing, 2002) show that positive GDP growth is not always correlated with greater bank profitability. In a study of Arabic banks, for example, stubbing (2002) finds a decline in earnings despite robust GDP growth. It appears that banks' performance is less likely to depend on the GDP growth rate if the banking industry is highly competitive with tighter margins and if the global investment markets are weak. Similarly, fund rate is found to have an impact on banks performance independent of other macroeconomic variables (Akhigbeand McNulty, 2005). By using both variables, our study shows that these two variables together could explain 12-15\% of the variation in bank's performance. Furthermore, the results pertaining to several determinant variables both confirm those of previous studies and extend them into a cross-regional context. For example, in a study of the profitability impacts of bank specific, industry, and macroeconomic variables in the Greek banking industry, Athanasoglou, Brissimis, and Delis (2008) find that increased exposure to credit risk lowers profits among Greek banks. They also note positive impacts of labor productivity (revenue/labor) and operational efficiency on profitability on bank performance. Goddard, Molyneux, and Wilson (2004), in studying European banks, identified capital-asset ratio (CAR) as positively affecting banks' performance for all countries. High CAR should signify that a bank is operating over-cautiously and ignoring potential profits.

Meanwhile, the differences in sales growth among U.S., Japanese, and Italian banks are best explained by the following variables: fund rate $(\beta=.60 ; p<.001)$, interest spread ratio $(\beta=.34 ; \mathrm{p}<.001)$, operational expenses/net sales $(\beta=-.22 ; \mathrm{p}<$ $.001)$, GDP growth $(\beta=.18 ; \mathrm{p}<.001)$, last year's performance $(\beta=.14 ; \mathrm{p}<.001)$, net sales/assets $(\beta=-.12 ; \mathrm{p}<.001)$, interest income/net sales $(\beta=-.07 ; \mathrm{p}<.001)$, common equity/assets $(\beta=-.06 ; p<.001)$, and loans/assets $(\beta=.03 ; \mathrm{p}<.001)$. These results reflect a wider set of variables affecting sales growth than existing studies that considered sales growth as a key performance variable (cf. Bodla and Verma, 2006). Another general finding from analyses of the cross-regional sample is the persistence of bank performance from one year to the next regardless of the profitability or sales growth measure used; this finding is consistent with but yet extends the existing finance literature to a more global setting (e.g., Berger et al., 2000; Goddard, Molyneux, and Wilson, 2004).

There were notable differences in performance determinants based on the types of performance measures used in the analyses (profitability versus growth). For 
example, while fund rate has a strong positive effect on sales growth performance, it does not impact ROE and exhibits a negative effect on ROA (cf. Kosmidou, et al., 2006). Furthermore, while interest income/net sales and net sales/assets have strong positive effects on ROA and ROE, they have significant negative effects on sales growth. These findings are consistent with those of Alam (2003) and Kosmidou, et al. (2006).

Research by Hasan, Koetter and Wedow (2009) has shown that improved operational efficiency by banks positively influences sales growth, especially for regional economies such as European countries. Although we confirm the significant relationship between operational efficiency and bank performance (Stubbing, 2002; Bodla and Verma, 2006), the relative impact of this factor on bank growth is considerably smaller than its influence on bank profitability.

\subsection{Similarities and Differences in Success Factors between Domestic and Global Competition}

The results from separate regression analyses for each country show the key determinants of firm performance as banks compete in their respective domestic markets. Collectively, these results show what it takes for a bank to achieve superior performance in the domestic competitive environment. While single-country studies of bank performance exist in large numbers, one of our primary interests in conducting this study was to observe how drivers for strong performance persist or change as banks move from domestic competition to global competition. To the best of knowledge, no other studies have specifically addressed this issue before. Next, we summarize the key findings to this effect.

Notably, four variables, namely operational expenses/net sales, interest spread ratio (NII/INCOME), interest income/net sales, and common equity/asset are significant determinants of bank profitability (both ROA and ROE) both in three individual countries and globally, all at the .001 significance level. Also, fund rate, common equity/asset, interest spread ratio (NII/INCOME), and operational expenses/net sales are significant determinants of sales growth regardless of the competitive setting (domestic or global). These findings highlight the importance of developing capabilities in these core areas at home before competing in the global environments.

While it is also important to note the universality of certain success factors in domestic versus global competition, we were most interested in noting differences in keys to success as banks move from domestic completion to global competition.

The most important finding to this effect is the role of HHI or industry concentration in influencing bank performance. While it is a significant determinant of bank performance (both profitability and growth) in most domestic settings, it is nonsignificant in all cross-regional comparisons. Previous studies have identified the positive effects of industry concentration and common equity/assets ratios on ROA. Haggestad (1977) and Chirwa (2003) in their research found that there was a longterm positive relationship between industry concentration and bank performance. On the other hand, Athanasoglou, Brissimis, and Delis (2008) did not observe a strong correlation between industry concentration and bank's performance. Similarly, Yildirim and Philippatos (2007) in their study of 11 Latin American banks found that concentration in banking markets does not necessarily lead to lower level of competition and higher bank performance. Also, Yildirim and Philippatos (2007) 
showed that bank returns are negatively linked to the degree of competition and, to a lower extent, to foreign bank participation; and that industry concentration affected bank profitability differently from country to country. It appears that industry concentration does not have similar effects on bank's performance on a global scale. Our finding suggests that the level of success a bank can achieve in global competition has little to do with the level of industry concentration in its home market.

Furthermore, while fund rate is the single most important determinant of sales growth for commercial banks in all three countries, it has very different effects on bank profitability in these countries. Specifically, it positively influences bank profitability in the US (on ROA: $\beta=.08, \mathrm{p}<.001$; on ROE: $\beta=.13, \mathrm{p}<.001$ ); has no influence on bank profitability in Japan; and negatively impacts bank profitability in Italy (on ROA: $\beta=-.17, p<.01$; on ROE: $\beta=-.12, p<.05$ ).

Another point of difference between success factors in domestic and global markets is the impact of home country's GDP growth rate on bank performance. It is a significant determinant of bank performance on the global scale regardless of the measure used; yet, it does not explain differences in profitability among U.S. banks and the variability in sales growth among Japanese banks.

Also, while net sales/asset has a significant positive effect on bank profitability at the global level and in both U.S. and Italy, it does not explain differences in bank profitability among Japanese banks. On the other hand, while net sales/assets increases sales growth in Japan $(\beta=.25, \mathrm{p}<.001)$, it does not have a significant impact on sales growth in Italy and has a negative impact on sales growth in the U.S. $(\beta=-.22, \mathrm{p}<.001)$.

\section{Conclusion}

While the issue of bank performance has received considerable scholarly attention in the past, existing research can be critiqued in terms of its focus on single countries (such as U.S. or Greece) or countries of the same region (such as Europe or Latin America), use of single or few performance measures (e.g., bank profitability as opposed to both profitability and sales growth), or limited scopes of determinants. Only a limited number of studies have sought to explain bank performance through joint employment of profitability and growth performance criteria, use of determinants spanning bank, industry, and country factor categories (cf. Athanasoglou et al., 2008), and in cross-regional studies. The limited scopes of performance measures, of determinant variables, and of regions make it difficult to compare findings across studies and turn research findings into practical managerial insights as banks increasingly compete on a global scale. By using three performance measures (with both profitability and growth indicators) and data collected over twenty years from more than two hundred commercial banks from three of the largest industrialized countries spanning three regions, our study attempts to identify variables that best determine successful performance in a global banking environment.

Our key contribution is the comprehensive nature of our study in terms of the number of regions, the number of banks, the long range of years, and the inclusive nature of our dependent variables (both profitability and growth measures) and independent variables (bank, industry, and country determinants). The cross-regional 
nature of our study enabled us to yield insights into determinants of bank performance uniquely important in a global setting. The use of multiple performance measures allowed us to verify results and enhance confidence in our findings.

Based on these results, banks' cross-regional profitability performance as measured by ROA and ROE, the two most critical performance measures, are affected by multiple factors including bank operational variables such as operating efficiency, debt/equity, product diversification, and productivity; balance sheet structure variables such as common equity/assets; and external factors such as GDP and fund rate. The differences in sales growth among U.S., Japanese, and Italian banks are best explained by fund rate, interest spread ratio, operational expenses/net sales, GDP growth, last year's performance, net sales/assets, interest income/net sales, common equity/assets, and loans/assets. Collectively, these results show what it takes for firms to achieve superior profitability and growth performance in the global banking environment.

Another general finding is the persistence of bank performance from one year to the next regardless of the profitability or sales growth measure used; this finding is consistent with but yet extends the existing finance literature to a more global setting (e.g., Berger et al., 2000; Goddard, Molyneux, and Wilson, 2004). The finding that external variables such as GDP and fund rate affect bank performance across the three regions adds important insight to the bank performance literature. While previous research has shown the effects of external variables such as inflation rate and cyclical output on bank profitability (Athanasoglou et al., 2008), the use of fund rate and GDP as determinant variables is a unique feature of our study as this combination has not been studied previously.

While single-country studies of bank performance exist in large numbers, one of our primary contributions was to show how drivers for strong performance persist or change as banks move from domestic competition to global competition. To the best of knowledge, no other studies have specifically addressed this issue before.

Notably, four variables, namely operational expenses/net sales, interest spread ratio (NII/INCOME), interest income/net sales, and common equity/asset are significant determinants of bank profitability (ROA and ROE) in three individual countries and globally. Also, fund rate, common equity/asset, interest spread ratio (NII/INCOME), and operational expenses/net sales are significant determinants of sale growth regardless of the competitive setting (domestic or global). These findings highlight the importance of developing capabilities in these core areas at home before competing in the global environments.

Meantime, we noted differences in keys to success as banks' competitive arena moves from domestic to global. For example, while HHI or industry concentration is a significant determinant of bank performance (both profitability and growth) in most domestic settings, it is non-significant in cross-regional comparisons. Furthermore, while fund rate is the single most important determinant of sales growth for commercial banks in all three countries, it has very different effects on bank profitability in three countries.

In addition to the above contributions, our results add to the existing literature on bank performance by illustrating the cross-country similarities and differences in the effects of a host of firm, industry, and country determinants on bank performance. For example, we note that three general findings consistent among the three studied countries were the persistence of bank performance from one year to the next, the 
importance of operational expenses/net sales as a performance determinant, and the lack of significance of asset as a bank performance predictor. These findings, while similar to those of Berger et al. (2000), Goddard, Molyneux, and Wilson (2004), Barros, Ferreira, and Williams (2007), and Kosmidou et al. (2006), are more extensive in revealing the differences in bank performance across three industrialized countries. We further use multiple performance measures (for both profitability and growth) to verify robustness of the findings and their unique determinants. In turn, we examine differences in effects of various determinants on bank performance. For example, GDP growth is more important as a predictor of bank performance in Japan than in both the United States and Italy, while net interest income/interest income is a more prominent bank performance determinant in the United States than in both Japan and Italy. Furthermore, fund rate is generally an important determinant of bank performance in the United States but is largely unrelated to bank performance in Japan and Italy. The finding that bank performance is not only affected by operational variables and industry variables but also by country differences shows the need for banks to modify their strategic approaches as they move into other markets.

Authors of future studies could seek to replicate the study to cover more countries and compare their findings with ours. In the meantime, bank executives should be mindful of country-specific performance determinants as they formulate a strategy for a particular market. Last, but not least, future research is encouraged to study the impacts of financial crisis on the performance of commercial banks (Degl'Innocenti et al., 2017).

\section{References}

1. Akhigbe A., \& McNulty, J. 2005. Profit efficiency sources and differences among small and large U.S. commercial banks. Journal of Economics and Finance, 29(3): 289-299.

2. Alam, I. 2003. Innovation strategy, process and performance in the commercial banking industry. Journal of Marketing Management, 19: 973-999.

3. Al-Tamimi, H.A.H., \& Lootah, A.M. 2007. Evaluating the operational and profitability efficiency of a UAE-based commercial bank. Journal of Financial Services Marketing, 11(4): 333-348.

4. Athanasoglou, P., Brissimis, S., \& Delis, M. 2008. Bank-specific, industryspecific and macroeconomic determinants of bank profitability. Journal of International Financial Markets, Institutions \& Money, 18(2): 121-136.

5. Barros, C.P., Ferreira, C., \& Williams, J. 2007. Analyzing the determinants of performance of best and worst European banks: A Mixed Logit approach. Journal of Banking and Finance, 31(12): 2189-2203.

6. Belsley, D., Kuh, E., \& Welsch, R. 1990. Regression diagnostics: Identifying influential data and sources of collinearity. New York, NY: Wiley.

7. Berger, A., Bonime, S., Covitz, D., \& Hancock, D. 2000. Why are bank profits so persistent? The roles of product market competition, informational opacity, and regional/macroeconomic shocks. Journal of Banking and Finance, 24(7): 1203-1235.

8. Bodla, B., \& Verma, R. 2006. Determinants of profitability of banks in India: a multivariate analysis. Journal of Services Research, 6(2): 75-89. 
9. Boulding, W., \& Staelin, R. 1995. Identifying generalizable effects of strategic actions on firm performance: The case of demand-side returns to R\&D spending. Marketing Science, 14(3): 222-234.

10. Bourke, P. 1989. Concentration and other determinants of bank profitability in Europe, North America, and Australia. Journal of Banking and Finance, 13(1): 65-79.

11. Bucevska, V., \& Misheva, B.H. 2017. The determinants of profitability in the banking industry: empirical research on selected Balkan countries. Eastern European Economies, 55, 146-167.

12. Canhoto, A., \& Dermine, J. 2003. A note on banking efficiency in Portugal: New vs. old banks. Journal of Banking and Finance, 27(11): 2087-2098.

13. Chine-Ta, H. 2006. Measuring bank performance: An approach based on grey relation analysis. Journal of the Operational Research Society, 57(4): 337-349.

14. Chirwa, E.W. 2003. Determinants of commercial banks' profitability in Malawi: A cointegration approach. Applied Financial Economics, 13(2003): 565-571.

15. Cornett, M.M., Ors, E., \&Tehranian, H. 2002. Bank performance around the introduction of a section 20 subsidiary. The Journal of Finance, 57(1): 501-521.

16. De Guevara, J.F., \& Maudos, J. 2002. Inequalities in the efficiency of the banking sectors of the European Union. Applied Economics Letters, 9(2002): 541-544.

17. Degl'Innocenti, M., Kourtzidis, S. A., Sevic, Z., \& Tzeremes, N. G. 2017. Bank productivity growth and convergence in the European Union during the financial crisis. Journal of Banking and Finance, 75, 184- 199.

18. Demirguc-Kunt, A., \& Huizinga, H. 1998. Determinants of commercial bank interest margins and profitability: Some international evidence. World Bank Economic Review, 13(2): 379-408.

19. Fadzlan, S., \&Majid, M-Z.A. 2007. Bank mergers performance and the determinants of Singapore banks' efficiency: An approach of two-stage banking models. International Journal of Business, 9(1): 19-39.

20. Ferris, S., Kumar, R., Sant, R., \& Sopariwala, P. 1998. An agency analysis of the effect of long term performance plan on managerial decision making. Quarterly Review of Economics and Finance, 38(1): 73-91.

21. Fraser, D., Phillips, W. Jr., \& Rose, P. 1974. A canonical analysis of bank performance. Journal of Financial and Quantitative Analysis, 9(2): 287-295.

22. Gintschel, A., \& Hackethal, A. 2004. Multi-bank loan pool contracts: Enhancing the profitability of small commercial banks. Applied Financial Economics, 14(17): 1239-1252.

23. Goddard, J., Molyneux, P., \& Wilson, J. 2004. The profitability of European banks: A cross-sectional and dynamic panel analysis. Manchester School, 72(3): 363-381.

24. Greene, W.H. 2003. Econometric analysis. 5th ed. Englewood Cliffs, NJ: Prentice Hall.

25. Griffith, J.M., Fogelberg, L., \& Weeks, H.S. 2002. CEO ownership, corporate control, and bank performance. Journal of Economics and Finance, 26(2): 170183.

26. Hartman, C. 2006. Data: Is more better...or is it simply more. Bank Accounting and Finance, 19(3): 39-42. 
27. Hasan, I., Koetter, M., \& Wedow, M. 2009. Regional growth and finance in Europe: Is there a quality effect of bank efficiency? Journal of Banking and Finance, 33 (8):1446-1453.

28. Heggestad, A. 1977. Market structure risk and profitability in commercial banking. The Journal of Finance, 32(4): 1207-1216.

29. Isik I., \& Hassan, M. 2002. Technical, scale and allocative efficiencies of Turkish banking industry. Journal of Banking and Finance, 26(4): 719-766.

30. Jensen, M.C., \& Meckling, W.H. 2009, Specific knowledge and divisional performance measurement, Journal of Applied Corporate Finance, 21(2): 49-57.

31. Kennedy, P. 2003. A guide to econometrics. 5th edition ed. Cambridge, MA: The MIT Press.

32. Kosmidou, K., Pasiouras, F., Zopounidis, C., \& Doumpos, M. 2006. A multivariate analysis of the financial characteristics of foreign and domestic banks in UK. Omega, 34: 189-195.

33. Kumar, R., \& Sopariwala P. 1992. The effect of adoption of long-term performance plans on stock prices and accounting numbers. Journal of Financial and Quantitative Analysis, 27(4): 561-575.

34. Mansur, I., \& Elyasiani, E. 1995. Sensitivity of bank equity returns to the level and volatility of interest rates. Managerial Finance, 21: 57-78.

35. Mehra, A. 1996. Resource and market based determinants of performance in the U.S. banking industry. Strategic Management Journal, 17(4): 307-322.

36. Mehra, S. 2006. A strategic review of operations and marketing functions in retail banks. International Journal of Service Industry Management, 17(3/4): 364-379.

37. Naceur, S., \& Goaied, M. 2001. The determinants of the Tunisian deposit banks' performance. Applied Financial Economics, 11(3): 317-319.

38. Nagar, V. \& Rajan, M. V. 2005. Measuring customer relationships: The case of retail banking industry. Management Science, 51(6): 904-919.

39. Ongore, O.K. 2013. Determinants of commercial banks performance in Kenya. International Journal of Economics and Financial Issues, 3 (1), 237-252.

40. Park, K.H., \& Weber W.L. 2006. Profitability of Korean banks: Test of market structure versus efficient structure. Journal of Economics and Business, 58(3): 222-239.

41. Quagliariello, Mario. 2009. Macroeconomic uncertainty and bank's lending decisions: The case of Italy. Applied Economics, 41(3): 323-336.

42. Ramsey, J.B. 1969. Tests for specification error in classical linear least squares regression analysis. Journal Royal Statistical Society Series B 31: 350-371.

43. Reilly, F.K., \& Wright, D.J. 2004, Analysis of risk-adjusted performance of global market assets. Journal; 30(3): 63-77.

44. Seemule, M., Sinha, N., \& Ndlovu, T. 2017. Determinants of commercial banks' profitability in Botswana: an empirical analysis. IUP Journal of Bank Management, 16(2), 7-28.

45. Sensarma, R. 2006. Are foreign banks always the best? Comparison of stateowned, private and foreign banks in India. Economic Modeling, 23(4): 717-735.

46. Shehzad, C.T., de Haan, J., \& Scholtens, B. 2013. The Relationship between Size, Growth and Profitability of Commercial Banks. Applied Economics. 45 (13), 1751-1765. 
47. Short, B. K. 1979. The relation between commercial bank profit rates and banking concentration in Canada, Western Europe, and Japan. Journal of Banking and Finance, 3: 209-219.

48. Staikouras, S. K. 2005. Equity returns of financial institutions and the pricing of interest rate risk. Applied Financial Economics, 15(7): 499-508.

49. Strischek, D. 1987. Return of the leveraged debtor: ROE versus ROA. Journal of Bank Lending, 69: 2-13.

50. Stubbing, D. 2002. Earnings fall at many Arab banks. Euro money. 401: 102107.

51. Wan, W.P., Yiu, D.W., Hoskisson, R.E., \& Kim, H. 2008. The performance implications of relationship banking during macroeconomic expansion and contraction: A study of Japanese banks social relationships and overseas expansion. Journal of International Business Studies. 39 (3): 406-427.

52. Wu, H.-L., Chen, C.-H., \& Lin, M.-H. 2007. The Effect of foreign bank entry on the operational performance of commercial banks in the Chinese transitional economy. Post-Communist Economies, 19(3): 343-357.

53. Yildirim, C. 2002. Evolution of banking efficiency within an unstable macroeconomic environment: the case of Turkish commercial banks. Applied Economics, 34: 2289-2301.

54. Yildirim, H.S., \& Philippatos, G.C. 2007. Restructuring, consolidation and competition in Latin American banking markets. Journal of Banking and Finance, 31(3): 629-639.

\section{About Our Authors}

Tao (Tony) GAO Ph.D., is an Associate Professor of Marketing at Manning School of Business, University of Massachusetts Lowell. He conducts research on the management of customer relationships, marketing innovations, and international marketing. He has published in Journal of Business Research, Journal of Business Ethics, Journal of Interactive Marketing, Journal of International Marketing, Journal of Service Research, Industrial Marketing Management, Journal of Advertising Research, Business Horizons, and Journal of Service Management, Multinational Business Review, among other journals.

James P. Neelankavil, Ph.D., is the Distinguished Professor Emeritus in the Department of Marketing and International Business at the Zarb School of Business of Hofstra University. He earned a MBA with distinction from the Asian Institute of Management, Manila, the Philippines and his Ph.D. in International Business from the Stern School of Business of New York University. Dr. Neelankavil has published over 30 articles in highly regarded refereed journals including in the Journal of International Business Studies (JIBS), Journal of Business Research (JBR), International Review of Applied Economics, and Journal of Advertising Research (JAR). In addition, he has published 8 books the areas of International Business and Corporate Strategy.

K.G. Viswanathan, Ph.D., is an Associate Professor and the Chairperson in the Department of Finance at the Zarb School of Business of Hofstra University. He earned an MBA from Western Michigan University, Kalamazoo, Michigan, and his 
Ph.D. in Finance from University of Tennessee, Knoxville, TN. His current areas of research include capital markets, cross-country investment diversification, and sustainability finance investing. His research has been published in journals such as Journal of Financial Research, Global Finance Journal, and Quarterly Review of Economics and Finance. 